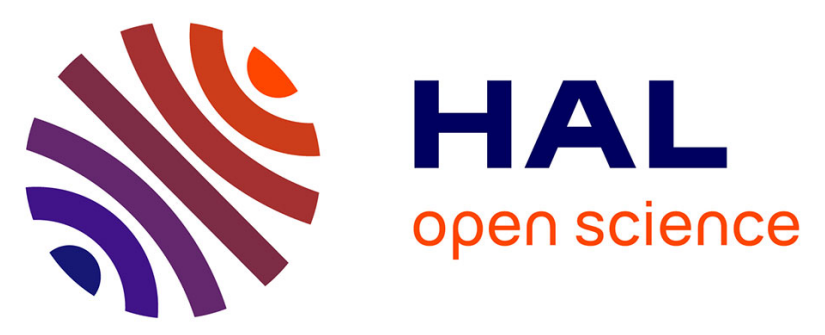

\title{
Numerical investigation of meniscus deformation and flow in an isothermal liquid bridge subject to high-frequency vibrations under zero gravity conditions
}

\author{
D.V. Lyubimov, T. P. Lyubimova, R.V. Skuridin, G. Chen, B. Roux
}

\section{- To cite this version:}

D.V. Lyubimov, T. P. Lyubimova, R.V. Skuridin, G. Chen, B. Roux. Numerical investigation of meniscus deformation and flow in an isothermal liquid bridge subject to high-frequency vibrations under zero gravity conditions. Computers and Fluids, 2002, 31, pp.663-682. 10.1016/S0045-7930(01)00078-0 . hal-01307320

\author{
HAL Id: hal-01307320 \\ https://hal.science/hal-01307320
}

Submitted on 29 Apr 2016

HAL is a multi-disciplinary open access archive for the deposit and dissemination of scientific research documents, whether they are published or not. The documents may come from teaching and research institutions in France or abroad, or from public or private research centers.
L'archive ouverte pluridisciplinaire HAL, est destinée au dépôt et à la diffusion de documents scientifiques de niveau recherche, publiés ou non, émanant des établissements d'enseignement et de recherche français ou étrangers, des laboratoires publics ou privés. 


\title{
Numerical investigation of meniscus deformation and flow in an isothermal liquid bridge subject to high-frequency vibrations under zero gravity conditions
}

\author{
D.V. Lyubimov ${ }^{\text {a }}$, T.P. Lyubimova ${ }^{\text {b }}$, R.V. Skuridin ${ }^{\text {b }}$, G. Chen ${ }^{c}$, B. Roux ${ }^{c}, *$ \\ ${ }^{a}$ Perm State University, 15, Bukireva Str., 614600 Perm, Russia \\ ${ }^{\mathrm{b}}$ Institute of Continuous Media Mechanics UB RAS, 1, Koroleva Str., 614013 Perm, Russia \\ ${ }^{\mathrm{c}}$ Laboratoire de Modłisation et Simulation Numærique en Meanique (L3M) FRE2405 du CNRS, \\ IMT La Jete-Technopole de Chateau-Gombert, 13451 Marseille Cedex 20, France
}

\begin{abstract}
This paper deals with meniscus deformation and flow in an isothermal liquid bridge maintained between two circular rods, when one rod is subject to axial monochromatic vibrations. It concerns a fundamental aspect of the problem of crystal growth from melt by the floating-zone technique which is often considered in weightlessness conditions. In the absence of vibrations the bridge is cylindrical; but due to vibration the mean shape of the meniscus is no more cylindrical and the meniscus oscillates around this mean shape. Two models are developed. First, we take into account the pulsating deformations of the meniscus (free surface), but we assume that the mean shape of meniscus remains cylindrical (i.e., we neglect the influence of vibration on this mean shape). For this simple case, a solution of the problem for the pulsating meniscus deformations and the pulsating velocity field is found in explicit form. For the mean flow, the problem is solved numerically by a finite-difference method. The calculations demonstrate the contribution of two basic mechanisms of mean flow generation due to vibrations, related to the generation of mean vorticity in the viscous boundary layer near the rigid boundaries and surface-wave propagation at a free surface. The intensity of the mean flow induced by surface waves is found to be sharply increasing when the vibration frequency approaches the resonance values that are determined from the explicit form of the solution of pulsation problem. In the second model, we take into account both pulsating and mean deformations of the meniscus. The governing equations for the potential of pulsating velocity and mean velocity, and for the pressure, are solved by using a finite-difference method and a boundary-fitted curvilinear coordinate system fitting the free surface.
\end{abstract}

Keywords: Isothermal liquid bridge; Microgravity; High-frequency vibrations; Numerical simulation

Computers \& Fluids 31, pp. 663-682 (2002) 


\section{Introduction}

A comparatively new technique to introduce defined flow structures or to control timedependent convective regimes in crystal growth from the melt is the vibration of the solid-liquid interface. Especially for growth arrangements with free melt surfaces like floating-zone growth, the vibrating boundary generates surface flows away from the interface and therefore can be used to counterbalance the flow due to the thermocapillary forces [1,2]. But the modeling of such a floating-zone growth is very complex, and it is important to firstly consider a more fundamental study of the vibration-driven flow inside an isothermal liquid bridge subject to vibrations. Nonlinear response of liquid bridges subjected to axial low-frequency vibrations is studied in Refs. [3,4]. One-dimensional non-linear viscous model is developed in Ref. [5] to study streaming generated in a long liquid bridge due to vibrations of an endwall. The present paper deals with the effects of high-frequency vibrations which are considered in Refs. [1,2] as most promising for a floating-zone growth of semi-conductor materials.

It is known that externally applied high-frequency vibrations exert significant influences on fluid systems with a free surface or interface (see, for example, Refs. [6,7]). To study the dynamics of systems subject to high-frequency vibrations it is convenient to decompose the flow field into mean and pulsating components and to obtain a closed set of equations for the mean flow. This approach has been used by Schlichting [8] for the flow near an oscillating cylinder, and by Longuet-Higgins [9] for the mean flow under surface waves in water. In both cases, the pulsating vorticity was found to have a large magnitude in a boundary layer. Non-linear interaction leads to the generation of a mean vorticity, which diffuses from the boundary layer to the bulk, due to viscosity. This effect has been extensively studied in the last years, however most of the works deal with the case of fluid system of infinite extent. The general approach for the description of vi-bration flows in a fluid with a deformable free surface of finite extent, subjected to high-frequency vibrations, has been developed in Refs. [10-12]. In Ref. [13] pulsating and mean flows in a liquid bridge were considered for the case when the two rigid rods supporting the bridge oscillate synchronously in axial direction; the pulsating deformation of the free surface was taken into account but not the mean deformation. In the present paper, we study the response of an iso- thermal liquid bridge to axial high-frequency vibrations of one supporting rod, accounting for both pulsating and mean deformations of the free surface.

\section{Problem formulation}

\subsection{Governing equations}

Consider an isothermal liquid bridge under zero gravity conditions. The liquid is maintained between two rigid rods of equal radius $\mathrm{R}$, located at the distance $\mathrm{L}$ from each other (Fig. 1). One of the rods performs monochromatic oscillations in the axial direction with the angular frequency $x$ and the amplitude a. The volume of liquid is such that in absence of vibration the bridge is a 


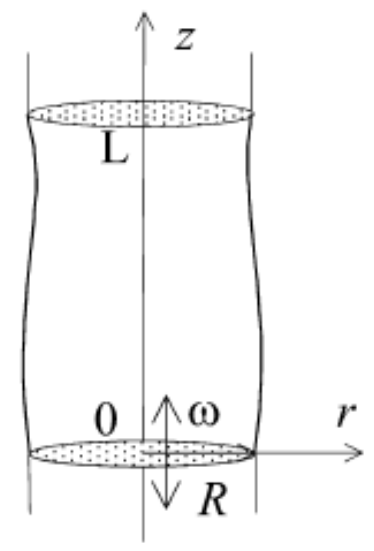

Fig. 1. Geometry of the problem.

circular cylinder of radius $R$ (at $g=0$ ). Due to vibrations, the mean shape of the liquid bridge can depart from cylindrical and the meniscus oscillates around its mean shape.

The Navier Stokes equations for an incompressible fluid are:

$$
\begin{aligned}
& \frac{\partial \vec{v}}{\partial t}+\vec{v} \cdot \nabla \vec{v}=\frac{1}{\rho} \nabla p+v \Delta \vec{v}_{1} \\
& \nabla \cdot \vec{v}=0
\end{aligned}
$$

where $\vec{v}$ is the velocity, $v$ is the kinematic viscosity and $p$ is the pressure.

In order to obtain an amenable set of equations, we make the following assumptions. First, we assume that the vibration period is much smaller than the viscous time scale, namely

$$
\omega^{-1} \ll R^{2} / v_{1}
$$

which also means that the thickness of the Stokes boundary layer $\delta=(v / \omega)^{1 / 2}$ is much less than a characteristic dimension of the liquid zone:

$$
\delta \ll R .
$$

Secondly, vibrations are not in an acoustic range, namely the sound wavelength at the vibration frequency is much greater than the characteristic dimension of the zone.

Thirdly and finally, the vibration amplitude is assumed to be small:

$$
a \ll R \text {. }
$$

These assumptions permit to decompose the hydrodynamical fields into the sums of a slowly varying (i.e., mean) and rapidly oscillating (i.e., pulsating) components:

$$
\begin{aligned}
& \vec{v}=\vec{u}+\vec{v}_{\mathrm{F} 1} \quad \overline{\vec{v}}_{\mathrm{F}}=0_{1} \quad \vec{u}=\overline{\vec{v}}_{1} \\
& p=\bar{p}+P_{1}
\end{aligned}
$$

where overbar denotes the average over the vibration period. 
Substituting Eqs. (2.6) and (2.7) into Eqs. (2.1) and (2.2) and retaining only the leading order terms in the equations for pulsations, we obtain the following equations

$$
\nabla \wedge \vec{v}_{\mathrm{F}}=0, \quad \nabla \cdot \vec{v}_{\mathrm{F}}=0
$$

for the pulsating fields, and the equations

$$
\begin{aligned}
& \frac{\partial \vec{u}}{\partial t}(\vec{u}+\vec{S}) \wedge(\nabla \wedge \vec{u})=\frac{1}{\rho} \nabla \vec{p}+v \Delta \vec{u}_{1} \\
& \nabla \cdot \vec{u}=0
\end{aligned}
$$

for the mean components where $\vec{S}=\overrightarrow{\vec{f} \cdot \nabla \vec{v}_{\mathrm{p}}}$ is the Stokes transport velocity, $\vec{f}$ is the vector determined by the formulae $\vec{v}_{\mathrm{F}}=(\partial \vec{f} / \partial t)$ and $\overrightarrow{\vec{f}}=0$, the sum $(\vec{u}+\vec{S})$ represents the Lagrangian velocity $\vec{u}_{1}$ (for details, see Ref. [12]).

As one can see, equations for the mean fields (2.9) and (2.10) differ from the conventional Navier Stokes equations by the additional term $\vec{S} \wedge(\nabla \wedge \vec{u})$. This term is responsible for the mean transport of vorticity due to the pulsating components of the flow. For monochromatic vibrations we have

$$
\vec{v}_{\mathrm{p}}=a \omega \operatorname{Re}\{\vec{V} \exp (\mathrm{i} \omega t)\}_{1}
$$

where $\vec{V}$ is the dimensionless complex-valued amplitude of the pulsating velocity, scaled with $a \omega$. In this case the Stokes transport velocity is

$$
\vec{S}=\frac{1}{2} a^{2} \omega \operatorname{Im}\left\{(\vec{V} \cdot \nabla) \vec{V}^{*}\right\} .
$$

\subsection{Boundary conditions for the mean velocity on the rigid boundaries}

As shown by Schlichting [8], the tangential components of the mean velocity can reach a finite value at the edge of the dynamical skin layers near the rigid surfaces where the generation of a mean vorticity occurs. For monochromatic vibrations with velocity pulsation having an inhomogeneous phase, the Schlichting boundary condition has the form $[12,15]$ :

$$
\vec{u}_{\tau}=\frac{a^{2} \omega}{2} \operatorname{Re}\left\{\frac{1}{2}\left(\vec{V}_{\tau} \cdot \nabla\right) \vec{V}_{z}^{*}+\left(1+\frac{3}{2} i\right) \vec{V}_{\tau}\left(\nabla \cdot \vec{V}_{\tau}^{*}\right)\right\} .
$$

Here derivatives are taken tangent to the boundary sufface, ${ }^{*}$ denotes the complex conjugate, $\vec{u}_{r}$ and $\vec{V}_{x}$ are the tangential components of vectors $\vec{u}$ and $\vec{V}$ on the rigid boundary.

Taking into account that the full mass transport through the boundaries should be equal to zero we obtain the following conditions for normal component of mean velocity on the rigid boundaries:

$$
u_{n}+S_{n}=0 \text {. }
$$

\subsection{Boundary conditions for the mean velocity on the free surface}

A vortex boundary layer adjacent to the free surface can be generated by the propagation of surface waves caused by the imposed vibration. An analysis of the boundary layer structure near 
the free surface shows that the mean shear rate near the free surface differs from zero [9]. For arbitrary sufface wave field, this leads to the following formula for the mean vorticity at the free surface $[12,16]$ :

$$
\nabla \wedge \vec{u}=a^{2} \omega \operatorname{Re}\left\{\nabla \zeta\left(\nabla \cdot \vec{V}^{*}\right)+\nabla \zeta \cdot \nabla \vec{V}^{*}\right\} \wedge \vec{n}_{1}
$$

where $\zeta$ is the dimensionless complex valued amplitude of the deviation of a free surface from its mean position (scaled with $a$ ) in the direction normal to this surface.

It follows from the condition of zero full mass transport through the boundaries that the mean velocity normal to the free surface $u_{n}$ differs from zero and is given by $[12,16]$ :

$$
u_{n}=\frac{1}{2} a^{2} \omega \operatorname{Re}\left\{\nabla \cdot\left(\zeta \vec{V}^{*}\right)\right\} .
$$

As shown in Ref. [17], the influence of mean flows on the mean shape of free surface is small in comparison with the mean effects of the pulsation action. That is why, in the mean nomal stress balance condition we can neglect the viscous stress term and write down this condition in the following form:

$$
\frac{\rho a^{2} \omega^{2}}{4}\left(\left|V_{n}\right|^{2} \quad\left|V_{z}\right|^{2}\right)+\sigma \nabla \cdot \vec{n}=C
$$

( $\vec{n}$ is the unit vector of external nomal to the free surface, $C$ is a constant). This condition, together with the condition on the contact lines define the mean shape of free surface.

Additionally we impose the condition of the liquid volume conservation.

\subsection{Boundary conditions for pulsations}

To implement the boundary conditions (2.13) and (2.14) we need to know the structure of the wave field $\zeta$ and the tangential components of the pulsating velocity near the free surface. Also, dissipative processes must be accounted for in order to avoid unthysical infinite growth of wave amplitude. If dissipative processes are considered, the flow will be non-potential. This makes the calculation of the pulsating fields more complicated. If, however, the penetration depth $\delta=(v / \omega)^{1 / 2}$ of the vortex component is small compared to that of the inviscid (or potential) component $\lambda / 2 \pi$ ( $\lambda$ is the wavelength of the surface waves), it is possible to use a quasi-potential approximation that considerably simplifies the analysis. Introducing complex amplitude of the potential components of the pulsating velocity $\vec{V}=\nabla \Phi$ we obtain respectively the kinematic condition and normal stress balance condition $[16,18]$ :

$$
\begin{gathered}
\omega^{2} \zeta \quad i \omega \frac{\partial \Phi}{\partial n}=2 v \nabla_{\tau}^{2} \frac{\partial \Phi}{\partial n}, \\
i \omega \Phi=\frac{\sigma}{\rho} \nabla_{\tau}^{2 \zeta}+2 v \nabla_{\tau}^{2} \Phi_{1}
\end{gathered}
$$

where $\nabla_{\tau}$ is the tangential part of the operator $\nabla$.

Additionally, we impose the stuck-edge conditions at the contact line:

$$
\left.\zeta\right|_{2=0}=\left.\zeta\right|_{2=L}=0 \text {. }
$$


The impermeability condition for the pulsating velocity are

$$
\left.\frac{\partial \Phi}{\partial n}\right|_{z=0}=\left.1_{1} \quad \frac{\partial \Phi}{\partial n}\right|_{z=L}=0
$$

for the vibrating and quiescent rods respectively.

No conditions for the tangential components of the pulsating velocity are imposed because the representation (2.6) and (2.7) and subsequent averaging reduces the order of the equations governing the pulsating part of the flow.

Note that surface-streaming effect occurs effectively only if the waves are propagating (i.e, not standing). In a float-zone configuration, propagating waves exist when there is a viscous damping. If the viscosity is not too low, the free-surface oscillation is not a system of standing waves but has a significant component corresponding to waves propagating from the oscillating rod. This promotes the effect of the fluid transport by the waves. At high viscosity, due to the strong wave damping, most of the free sufface will be at rest and the surface-streaming effect will be weak. One would expect that the optimal condition for mean-flow generation by surface waves is such that the length of wave damping, $l_{\mathrm{d}}$, is of the same order of magnitude as the size of the liquid bridge. It is known that for small dissipation, $l_{\mathrm{d}}$ is equal to the ratio of the wave group velocity to the decrement of wave damping [14]. For short enough waves, the cross-section curvature of the free surface should not be important; thus, for a crude estimate, we can use the dispersion relation for capillary waves on a flat surface; i.e., $l_{\mathrm{d}} \approx 3 \sigma /(4 \rho v \omega)$.

\subsection{Dimensionless equations and boundary conditions}

Let us restrict outselves to axisymmetric solutions and introduce cylindrical coordinate system with the origin located at the lower rod. By introducing the quantities $R, R^{2} / v, v / R, \rho v^{2} / R^{2}$ as the scales for the length, time, velocity and pressure we come to the following problem for the determination of the pulsating velocity field and pulsating deformations of the free surface, in dimensionless form:

$$
\frac{\partial^{2} \Phi}{\partial r^{2}}+\frac{1}{\gamma} \frac{\partial \Phi}{\partial r}+\frac{\partial^{2} \Phi}{\partial z^{2}}=0
$$

with the impermeability conditions for the pulsating velocity at the rigid rods

$$
\left.\frac{\partial \Phi}{\partial z}\right|_{z=0}=\left.1_{1} \quad \frac{\partial \Phi}{\partial z}\right|_{z=l}=0,
$$

the symmetry condition at the liquid bridge axis:

$$
\left.\frac{\partial \Phi}{\partial r}\right|_{r=0}=0
$$

and the following conditions at the free surface, at $r=f(z)$ :

$$
\mathrm{i} \Phi=W e^{-1} \nabla_{\tau}^{2} \zeta+2 \Omega^{-1} \nabla_{\tau}^{2} \Phi
$$




$$
\begin{aligned}
& \text { i } \zeta=\frac{\partial \Phi}{\partial n} \quad 2 i \Omega^{-1} \nabla_{z}^{2} \frac{\partial \Phi}{\partial n} \\
& \left.\zeta\right|_{z=0}=\left.\zeta\right|_{z=l}=0 .
\end{aligned}
$$

Dimensionless equations for the mean components read:

$$
\begin{aligned}
& \frac{\partial \vec{u}}{\partial t} \quad(\vec{u}+\vec{S}) \wedge(\nabla \wedge \vec{u})=\nabla \vec{p}+\Delta \vec{u}_{1} \\
& \nabla \cdot \vec{u}=0
\end{aligned}
$$

where

$$
\vec{S}=\frac{1}{2} R e_{\mathrm{F}} \operatorname{Lm}\left\{(\nabla \Phi, \nabla) \nabla \Phi^{*}\right\} .
$$

The boundary conditions for the mean components at the rigid rods, at $z=0, l$ are:

$$
\begin{aligned}
& u_{r}=\operatorname{Re}_{\mathrm{p}}\left[\frac{3}{4} \operatorname{Re}\left\{(1+1) \frac{\partial \Phi}{\partial r} \frac{\partial^{2} \Phi^{*}}{\partial r^{2}}\right\}+\frac{1}{2 r}\left|\frac{\partial \Phi}{\partial r}\right|^{2}\right] . \\
& u_{z}+S_{z}=0
\end{aligned}
$$

at the free surface, at $r=f(z)$ :

$$
\begin{aligned}
& \frac{\partial u_{\tau}}{\partial n} \quad \frac{\partial u_{n}}{\partial \tau}=2 \operatorname{Re} e_{\mathrm{p}} \operatorname{Re}\left\{\frac{\partial \zeta}{\partial \tau} \frac{\partial^{2} \Phi^{*}}{\partial \tau^{2}}\right\} 1 \\
& u_{n}+S_{n}=0_{1} \\
& B\left(\left|\frac{\partial \Phi}{\partial n}\right|^{2} \quad\left|\frac{\partial \Phi}{\partial \tau}\right|^{2}\right)+\nabla \cdot \vec{n}=C_{1} \\
& f(0)=1_{1} \quad f(l)=1
\end{aligned}
$$

at the symmetry axis, at $r=0$ :

$$
u_{r}=0, \quad \frac{\partial u_{2}}{\partial r}=0
$$

and the condition of the conservation of the liquid volume:

$$
\int_{0}^{l} f^{2}(z) d z=l
$$

Here $B=\rho a^{2} \omega^{2} R /(4 \sigma), l=L / R, i$ is the imaginary unit, $R e_{\mathrm{p}}=a^{2} \omega^{2} / v$ is the pulsating Reynolds number, $W e=\rho \omega^{2} R^{3} / \sigma$ and $\Omega=\omega R^{2} / v$ are, respectively, the Weber number and the dimensionless vibration frequency. The Ohnesorge number $O h=v[\rho /(R \sigma)]^{1 / 2}$ is related to $W e$ and $\Omega$ through the relation $O h=W e^{1 / 2} / \Omega$. Parameter $B$ is not an independent dimensionless parameter: it represents the combination $B=R e_{\mathrm{p}} W e /(4 \Omega)$. 


\section{Pulsating and mean flows in a liquid zone with non-deformable mean shape}

\subsection{Solution for pulsations}

The problem for the pulsating deformation of free sufface and the pulsating velocity field is decoupled from the mean-flow determination and can be solved independently. Let us assume that in the absence of vibration the liquid bridge is cylindrical, and neglect the vibration influence on the mean shape. For this simple case, it is possible to obtain the solution of the problem for the pulsations in explicit form. To this end, we represent the potential $\Phi$ in the following form:

$$
\Phi=\frac{1}{4 l} r^{2} \quad \frac{1}{2 l} z^{2}+z+\sum_{n=1}^{\infty} a_{n} I_{0}\left(f_{n} r\right) \cos \hbar_{n} z+\alpha_{1}
$$

which satisfies the boundary conditions on the rigid rods. Here $k_{n}=n \pi / l$ and $I_{0}$ is the modified Bessel function of zeroth-order.

Substituting Eq. (3.1) into the normal stress balance condition (2.23) we obtain a differential equation for the pulsating deviation of the free surface from its equilibrium position $\zeta$. The solution of this equation is:

$$
\zeta=\mathrm{i} W_{e}\left\{\frac{1}{2 l} z^{2}+z+\frac{5}{4 l}+\alpha \quad \frac{2 \mathrm{i}}{\Omega l}+\sum_{n=1}^{\infty} a_{n} \frac{\mathrm{l} 2 \mathrm{i} \hat{H}_{n}^{2} / \Omega}{\mathrm{l} \quad f_{n}^{2}} I_{0}\left(h_{n}\right) \cos \hbar_{n} z \quad F \frac{\sin z}{\sin l} \quad q \cos z\right\} .
$$

The formulas (3.1), (3.2) for $\Phi$ and $\zeta$ contain the constants $a_{n}, F, q$ and $\alpha$ that can be found from the kinematic condition (2.24) and stuckedge conditions (2.25):

$$
\begin{aligned}
& a_{n}=W e\left(\frac{2}{l} \frac{1}{k_{n}^{2}}+F \frac{2}{l \sin l} \frac{1}{1} \frac{(1)^{n} \cos l}{h_{n}^{2}}+2 q \frac{\sin l}{l} \frac{(1)^{n}}{1}\right) \\
& \times\left(\left(1+\frac{2 i}{\Omega} f_{n}^{2}\right) I_{0}^{\prime}\left(k_{n}\right) k_{n}+W e \frac{12 \mathrm{i} k_{n}^{2} / \Omega}{\mathbf{l} \frac{k_{n}^{2}}{2}} I_{0}\left(\mathfrak{k}_{n}\right)\right)^{-1} \\
& F=\frac{D_{F}}{D}, \quad q=\frac{D_{q}}{D}, \quad \alpha=\frac{2 \mathrm{i}}{\Omega l} \quad \frac{5}{4 l} \quad A_{1} \quad F A_{2} \quad q\left(A_{3} \quad 1\right)
\end{aligned}
$$

with

$$
\begin{aligned}
& D=\left(\begin{array}{lll}
\frac{\cos l}{l \sin l}+A_{2}
\end{array}\right)\left(\begin{array}{lll}
A_{3} & A_{6} & \mathrm{l}+\cos l)
\end{array}\left(\begin{array}{ll}
A_{2} & A_{5}+\mathrm{l}
\end{array}\right)\left(\begin{array}{ll}
\frac{\sin l}{l}+A_{3} & 1
\end{array}\right)\right. \\
& D_{F}=\left(\begin{array}{ll}
\frac{l}{3}+\frac{1}{2 l W e} & A_{1}
\end{array}\right)\left(\begin{array}{lll}
A_{3} & A_{6} & 1+\cos l)
\end{array}\left(\begin{array}{ll}
\frac{l}{2}+A_{4} & A_{1}
\end{array}\right)\left(\begin{array}{ll}
\frac{\sin l}{l}+A_{3} & 1
\end{array}\right)\right. \text {. } \\
& D_{q}=\left(\frac{1}{l \cos l}+A_{2}\right)\left(\frac{l}{2}+A_{4} \quad A_{1}\right) \quad\left(\begin{array}{ll}
A_{2} & A_{5}+1
\end{array}\right)\left(\begin{array}{ll}
\frac{l}{3}+\frac{1}{2 l W e} & A_{1}
\end{array}\right)
\end{aligned}
$$


where

$$
\begin{aligned}
& A_{1}=\frac{2}{l} S_{1_{1}} \quad A_{2}=\frac{2}{l \sin l}\left(S_{3} \quad S_{4} \cos l\right)_{1} \quad A_{3}=\frac{2}{l} S_{4} \sin l_{1} \quad A_{4}=\frac{2}{l} S_{2_{1}} \\
& A_{5}=\frac{2}{l \sin l}\left(S_{4} \quad S_{3} \cos l\right), \quad A_{6}=\frac{2}{l} S_{3} \sin l_{1} \quad S_{1}=\sum_{n=1}^{\infty} \frac{1}{k_{n}^{2} G_{n}}, \quad S_{2}=\sum_{n=1}^{\infty} \frac{(1)^{n}}{k_{n}^{2} G_{n}},
\end{aligned}
$$

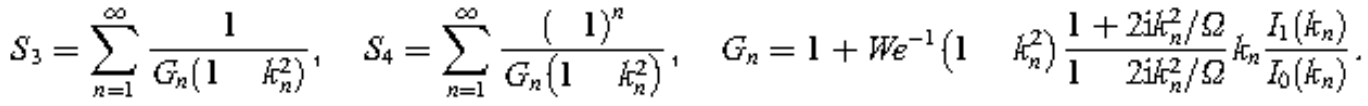

Thus, we obtain the solution of the problem (2.20) (2.25) for the pulsating velocity field and free surface pulsations, in explicit form. 25 lowest resonance values of the Weber number ate given in Table 1 for two aspect ratio values: $l=1$ and $l=2$.

\subsection{Solution for the mean flow}

Applying the operator $(\nabla \wedge)_{\varphi}$ to the mean flow equations and introducing the streamfunction and vorticity

Table 1

Resonance values of weber number for $l-1$ and $l-2$

\begin{tabular}{lll}
\hline$n$ & $W e, l-1$ & $W e, l-2$ \\
\hline 1 & $0.12155 \mathrm{E}+03$ & $0.10660 \mathrm{E}+02$ \\
2 & $0.52225 \mathrm{E}+03$ & $0.56600 \mathrm{E}+02$ \\
3 & $0.14357 \mathrm{E}+04$ & $0.16378 \mathrm{E}+03$ \\
4 & $0.29949 \mathrm{E}+04$ & $0.35260 \mathrm{E}+03$ \\
5 & $0.54388 \mathrm{E}+04$ & $0.64859 \mathrm{E}+03$ \\
6 & $0.89008 \mathrm{E}+04$ & $0.10728 \mathrm{E}+04$ \\
7 & $0.13619 \mathrm{E}+05$ & $0.16507 \mathrm{E}+04$ \\
8 & $0.19728 \mathrm{E}+05$ & $0.24033 \mathrm{E}+04$ \\
9 & $0.27466 \mathrm{E}+05$ & $0.33560 \mathrm{E}+04$ \\
10 & $0.36966 \mathrm{E}+05$ & $0.45300 \mathrm{E}+04$ \\
11 & $0.48466 \mathrm{E}+05$ & $0.59507 \mathrm{E}+04$ \\
12 & $0.62101 \mathrm{E}+05$ & $0.76391 \mathrm{E}+04$ \\
13 & $0.78109 \mathrm{E}+05$ & $0.96207 \mathrm{E}+04$ \\
14 & $0.96623 \mathrm{E}+05$ & $0.11917 \mathrm{E}+05$ \\
15 & $0.11788 \mathrm{E}+06$ & $0.14552 \mathrm{E}+05$ \\
16 & $0.14202 \mathrm{E}+06$ & $0.17549 \mathrm{E}+05$ \\
17 & $0.16927 \mathrm{E}+06$ & $0.20931 \mathrm{E}+05$ \\
18 & $0.19978 \mathrm{E}+06$ & $0.24721 \mathrm{E}+05$ \\
19 & $0.23377 \mathrm{E}+06$ & $0.28943 \mathrm{E}+05$ \\
20 & $0.27139 \mathrm{E}+06$ & $0.33620 \mathrm{E}+05$ \\
21 & $0.31287 \mathrm{E}+06$ & $0.38775 \mathrm{E}+05$ \\
22 & $0.35834 \mathrm{E}+06$ & $0.44431 \mathrm{E}+05$ \\
23 & $0.40805 \mathrm{E}+06$ & $0.50613 \mathrm{E}+05$ \\
24 & $0.46212 \mathrm{E}+06$ & $0.57341 \mathrm{E}+05$ \\
25 & $0.52080 \mathrm{E}+06$ & $0.64642 \mathrm{E}+05$ \\
\hline
\end{tabular}




$$
u_{r}=\frac{1}{r} \frac{\partial \psi}{\partial z}, \quad u_{2}=\frac{1}{\gamma} \frac{\partial \psi}{\partial r}, \quad \phi=\frac{1}{r}\left(\frac{\partial^{2} \psi}{\partial r^{2}} \frac{1}{\gamma} \frac{\partial \psi}{\partial r}+\frac{\partial^{2} \psi}{\partial z^{2}}\right)
$$

we obtain:

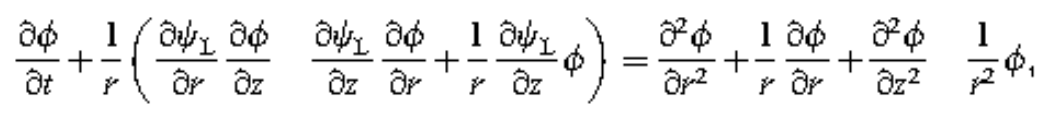

where $\psi_{1}=\psi+\psi_{s}, \psi_{s}$ is the streamfunction of the vector field $\vec{S}$ defined as:

$$
\psi_{\mathrm{s}}=\frac{1}{2} \operatorname{Re}_{\mathrm{p}} \operatorname{Im}\left\{r \frac{\partial \Phi}{\partial r} \frac{\partial \Phi^{*}}{\partial z}\right\} .
$$

The conditions for mean velocity on the rigid rods, at $z=0$, $l$, becomes:

$$
\psi_{\perp}=0, \quad \frac{\partial \psi}{\partial z}=\operatorname{Re}_{\mathrm{F}^{r}}\left[\frac{3}{4} \operatorname{Re}\left\{(1+1) \frac{\partial \Phi}{\partial r} \frac{\partial^{2} \Phi^{*}}{\partial r^{2}}\right\}+\frac{1}{2 r}\left|\frac{\partial \Phi}{\partial r}\right|^{2}\right]
$$

and on the free surface, at $r=1$ :

$$
\psi_{\perp}=0, \quad \phi=2 \operatorname{Re} e_{\mathrm{F}} \operatorname{Re}\left\{\frac{\partial \zeta}{\partial z} \frac{\partial^{2} \Phi^{*}}{\partial z^{2}}\right\}
$$

The problem (3.5) (3.7) is solved by a finite-difference method using ADI scheme.

\subsection{Numerical results}

To study the effect of vibration frequency, the calculations were carried out with the three nondimensional parameters $\left(R e_{\mathrm{p}}\right.$, We and $\Omega$ ) varying in such a way that the ratios $R e_{\mathrm{p}} / \Omega-(a / R)^{2}$ and $W e^{1 / 2} / \Omega-O h$ remain constant. We selected the values $a / R=0.003$ and $O h=2.934 \times 10^{-4}$, which correspond to a liquid metal with $\eta=0.88 \times 10^{-3} \mathrm{~g} \mathrm{~cm}^{-1} \mathrm{~s}^{-1}$ and $\sigma=720 \mathrm{dyn} \mathrm{cm}^{-1}$, with $R=0.5$ $\mathrm{cm}$ and $L=1 \mathrm{~cm}$ (i.e., $l=2$ ). The vibration amplitude is so small that both the vibration amplitude itself and the surface-wave amplitude are small in comparison with the characteristic size $R$. The maximum value of the Lagrangian streamfunction $\psi_{\perp}$ is chosen as characteristic of the flow intensity. The calculations show that $\max _{r_{2},}\left|\psi_{\perp}\right|$ sharply increases near the resonance values (Fig. 2). The flow intensity in areas between resonances gradually increases with the increase of $W e$, which is related to the decrease of the damping length, i.e. with the growth of the propagating component of the waves.

The only dimensionless parameter characterizing the vibration amplitude in isothermal problems is the pulsating Reynolds number (which is proportional to $a^{2}$ ). The calculations show that the mean flow intensity at a fixed value of $W e$ and fixed frequency (out of the resonance zones), increases almost linearly with $a^{2}$ (see Fig. 3). This demonstrates the possibility to get a strong 


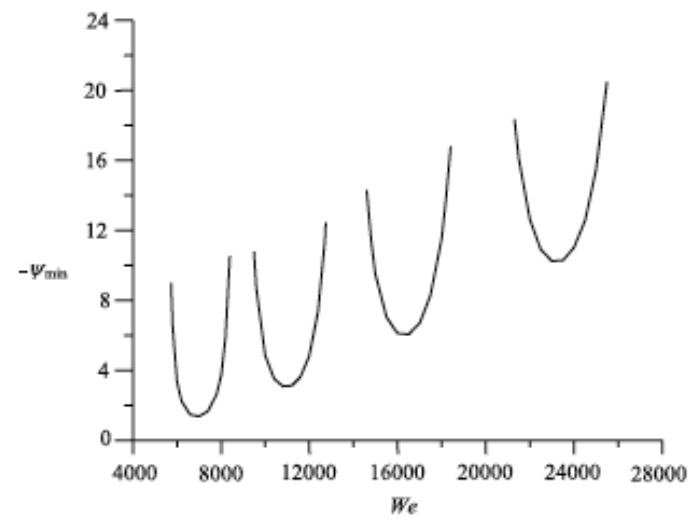

Fig. 2. Dependence of minimal value of streanfunction on $W e$, for $l-1$ and $a / R-0.003$.

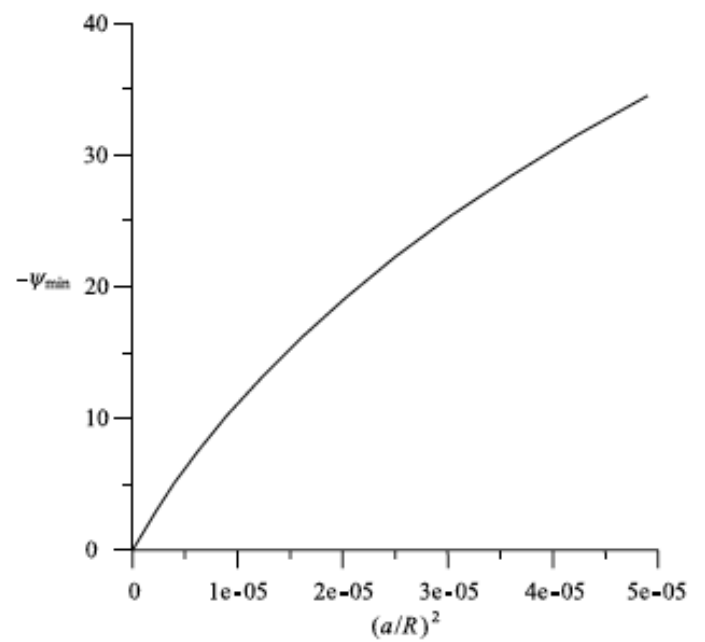

Fig. 3. Vibration amplitude dependence of minimal value of streamfunction, for $l-1$ and We -23000 .

enough vibration-induced flow, with dominating near-surface vortex, circulating along the free surface from the vibrating rod.

\section{Mean deformation and flow in a liquid bridge with a deformable free surface}

In this section, we consider pulsating and mean flows in the liquid bridge accounting for mean deformation of the free surface. In this case, the pulsation problem (2.20) (2.25) does not admit analytical solution and must be solved numerically. Also, the froblem is now coupled; so, we must consider the full problem (2.20) (2.36). 


\subsection{Numerical method}

\subsubsection{Grid generation}

Let us consider an irregular domain in the physical plane $(x, y)$ and use a boundary-fitted curvilinear coordinate system. Following Thompson et a1. [19], the Poisson equations

$$
\begin{aligned}
& \xi_{x x}+\xi_{y y}=P_{1} \\
& \eta_{x x}+n_{y}=Q
\end{aligned}
$$

give a curvilinear coordinates $(\xi, \eta)$ such that the irregular domain transforms into a rectangular one in the computational plane, i.e., $\xi_{\min } \leqslant \xi \leqslant \xi_{\max }, \eta_{\min } \leqslant n \leqslant \eta_{\max }$. After interchanging the dependent and independent variables, one obtains

$$
\begin{array}{ll}
\alpha x_{\xi \xi} & 2 \beta x_{\xi \eta}+\gamma x_{\eta \eta}=J^{2}\left(P x_{\xi}+Q x_{\eta}\right)_{1} \\
\alpha y_{\xi \xi} & 2 \beta y_{\xi \eta}+\gamma y_{\eta \eta}=J^{2}\left(B y_{\xi}+Q y_{\eta}\right)_{1}
\end{array}
$$

where

$$
\alpha=x_{\eta}^{2}+y_{\eta 1}^{2} \quad \beta=x_{\xi} x_{\eta}+y_{\xi} y_{\eta 1} \quad \gamma=x_{\xi}^{2}+y_{\xi 1}^{2} \quad J=x_{\xi} y_{\eta} \quad x_{\eta} y_{\xi} .
$$

The non-homogeneous terms $P$ and $Q$ are the control functions. Following Thompson et al, the values of $P$ and $Q$ inside the computational domain are computed by formulae [19]:

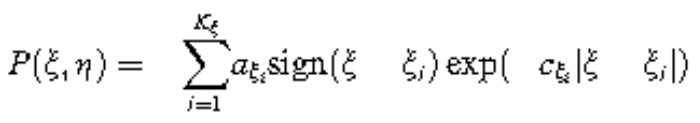

$$
\begin{aligned}
& \left.\sum_{j=1}^{L} b_{\xi_{j}} \operatorname{sign}\left(\xi \quad \xi_{j}\right) \exp \left(d_{\xi_{j}}\left[\begin{array}{ll}
\xi & \xi_{j}
\end{array}\right)^{2}+\left(\begin{array}{ll}
\eta & \eta_{j}
\end{array}\right)^{2}\right]^{1 / 2}\right)_{1} \\
& Q(\xi, \eta)=\sum_{j=1}^{K_{\eta}} a_{\eta_{i}} \operatorname{sign}\left(\eta \quad \eta_{j}\right) \exp \left(\begin{array}{cc}
c_{\eta_{i}} \mid \eta & \eta_{j}
\end{array}\right)
\end{aligned}
$$

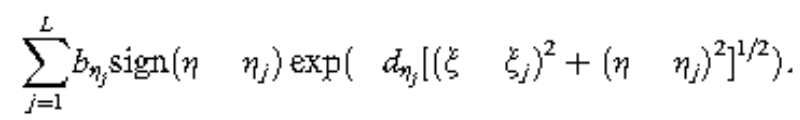

Here $K_{\xi}$ and $K_{\eta}$ stand for the line numbers $\xi=\xi_{j}$ and $\eta=\eta_{j}$, near which the grid contraction is desired, $L$ is the number of such points $\left(\xi_{j 1} \eta_{j}\right)$. In out case $\xi_{\min }=0, \xi_{\max }=1, \eta_{\text {rin }}=0$, $\eta_{\max }=\left(\begin{array}{lll}N Y & 1\end{array}\right) /\left(\begin{array}{ll}N X & 1\end{array}\right), K_{\xi}=1, K_{\eta}=2, L=0, \xi_{1}=1, \eta_{1}=0, \eta_{2}=\eta_{\max }, N X$ is the number of mesh points in $r$-direction, and $N Y$ in $y$-direction.

The associated boundary conditions are determined from the equations: on the lower and upper boundary

$$
x_{\xi \xi}=x_{\xi}^{3} P(\xi, 0), \quad x(0)=0, \quad x(1)=R_{1} \quad y(\xi)=0
$$

or

$$
x_{\xi \xi}=x_{\xi}^{3} P\left(\xi_{1} \eta_{\max }\right)_{1} \quad x(0)=0, \quad x(1)=R_{1} \quad y(\xi)=l_{1}
$$


on the symmetry axis

$$
y_{\eta \eta}=y_{\eta}^{3} Q(0, \eta), \quad y(0)=0, \quad y\left(\eta_{\max }\right)=l, \quad x(\eta)=0 ;
$$

on the liquid gas interface

$$
y_{\eta \eta}=y_{\eta}^{3} Q(\mathbf{1}, \eta), \quad y(0)=0, \quad y\left(\eta_{\max }\right)=l, \quad x=f(y) .
$$

The function $f(y)$ is supposed to be known. Since it is determined only in the grid nodes used in the previous iteration (or as initial approximation), a cubic spline interpolation is employed.

The non-linear Eqs. (4.8) (4.11) are discretized by a second-order centered finite-difference approximation and solved by Newton's method; the resulting three-diagonal linear system is solved by the DGTSV routine of LAPACK library. The Poisson-like Eqs. (4.3) and (4.4) are discretized in the same manner and solved by the successive over-telaxation (SOR) method.

In Eqs. (4.1) (4.11), the coordinate $x$ corresponds to $r$ of the Section 2.3, and $y$ to $z$. The expressions for the first derivatives of a field $\varphi$ are:

$$
\begin{aligned}
& \varphi_{x}=\left(\begin{array}{ll}
y_{\eta} \varphi_{\xi} & y_{\xi} \varphi_{\eta}
\end{array}\right) / J \\
& \varphi_{y}=\left(\begin{array}{ll}
x_{\xi} \varphi_{\eta} & x_{\eta} \varphi_{\xi}
\end{array}\right) / J .
\end{aligned}
$$

The Laplace operator in the cylindrical coordinate system takes the form:

$$
\varphi_{x x}+\varphi_{y y}+\frac{\varphi_{x}}{x}=\frac{\alpha \varphi_{\xi \xi} 2 \beta \varphi_{\xi \eta}+\gamma \varphi_{m}}{J^{2}}+\left(P+\frac{y_{\eta}}{x J}\right) \varphi_{\xi}+\left(Q \frac{y_{\xi}}{x J}\right) \varphi_{\eta} .
$$

The derivatives on the free surface take the form:

$$
\begin{aligned}
& \frac{\partial \varphi}{\partial n}=\frac{1}{J \sqrt{\alpha}}\left(\begin{array}{ll}
\alpha \varphi_{\xi} & \beta \varphi_{\eta}
\end{array}\right)_{1} \\
& \frac{\partial \varphi}{\partial \tau}=\frac{\varphi_{\eta}}{\sqrt{\alpha}} .
\end{aligned}
$$

The 'flat' Laplacian is approximated by

$$
\Delta_{2 \varphi} \approx \frac{\gamma \varphi \varphi_{m}}{J^{2}}+Q \varphi_{\eta}
$$

The expressions for the second and thitd derivatives can be obtained by using the general rules of variable change, but they are not given here for simplicity.

\subsubsection{Numerical algorithm}

All the derivatives are approximated by second-order central differences in the interior domain, and by second-order forward or backward differences on the boundaries. The velocity components, the potential of pulsating flow, the functions $f$ and $\zeta$ are defined on the grid points, and the pressure in the mesh center.

The computation of mean flow field is decoupled from the problem of determination of pulsating flow and liquid gas interface shape. So, after discretization we obtain a linear system of equations for $\Phi$ and $\zeta$, a non-linear system for $f$ (and for a constant $C$ ) which, in its turn, 
depends on the shape of the computational domain and the used grid, and a non-linear system for $u_{r}, u_{2}, p$.

To solve non-linear systems, Newton's method is applied. For a non-linear algebraic system written in vector form: $\mathbf{f}(\mathbf{X})=0$, where $\mathbf{X}$ is the unknown vector, we have:

$$
\begin{aligned}
& \mathbf{f}_{X}\left(\mathbf{X}^{n}\right) \mathrm{d} \mathbf{X}^{n}=\mathbf{f}\left(\mathbf{X}^{n}\right)_{1} \\
& \mathbf{X}^{n+1}=\mathbf{X}^{n}+\mathrm{d} \mathbf{X}^{n}{ }_{1}
\end{aligned}
$$

where $\mathbf{f}_{X}=\partial \mathbf{f} / \partial \mathbf{X}$ is the Jacobian matrix. The iterations continue until some convergence criterion is attained.

The associated Jacobian matrices are banded (except for the case of mean shape, in which the constant $C$ is present in equations for any interior point). The band LU factorization and the resolution of triangular systems are performed by using the CGBFA/CGBSL sequences in the LINPACK library.

The system for $(f, C)$ it formed in the natural way: line $j$ of matrix corresponds to the point $j$, but with supplemental equation number $N Y+1$. The solution is obtained by the use of routines DGEFA/DGESL of LINPACK library.

The values of $u_{r}, u_{2}, p$ for each node are arranged sequentially in the unknown vector $\mathbf{X}$. The resulting system is solved by the DGBF/DGBS routines of LINPACK library. The solution procedure is summatized as follows:

1. provide an initial guess of the solution;

2. transform the physical domain into a rectangular computational domain;

3. solve equations for $\Phi$ and $\zeta$;

4. solve equations for $u_{r}, u_{2}, p$;

5. determine a new free surface shape.

The steps 25 are repeated until the prescribed convergence criteria are satisfied. Then the appropriate Poisson equation for the streamfunction $\psi$ is solved by using the computed velocity field, and the transition to the Lagrangian streamfunction is made.

\subsection{Numerical results}

The computations were performed on the grid $(N X=41) \times(N Y=151)$, for the following values of the dimensional parameters: rod radius $R=0.5 \mathrm{~cm}$, zone length $L=1.0 \mathrm{~cm}$, density $\rho=2.53 \mathrm{~g} \mathrm{~cm}^{3}$, dynamic viscosity $\eta=8.855 \times 10^{-3} \mathrm{~g} \mathrm{~cm}^{-1} \mathrm{~s}^{-1}$, surface tension coefficient $\sigma=$ $720 \mathrm{dyn}_{\mathrm{cm}}^{-1}$, vibration amplitude $a=1.5 \times 10^{-3} \mathrm{~cm}$, vibration frequency was varied in the range $10^{2} \quad 1.23 \times 10^{4} \mathrm{~s}^{-1}$.

Fig. 4a and b show typical views of the pulsating velocity potential and of the pulsating deformation of the free surface. It can be seen that the real part of the pulsating deformation is small and decreases with the increase of distance from the vibrating rod, showing the establishment of the standing wave regime, in which the wave propagating from the vibrating rod has nearly the same amplitude as the wave reflected from the quiescent rod. As a result, the mean flow generation near the free surface leads to the formation of a large number of small vortices whose di- 
(a)

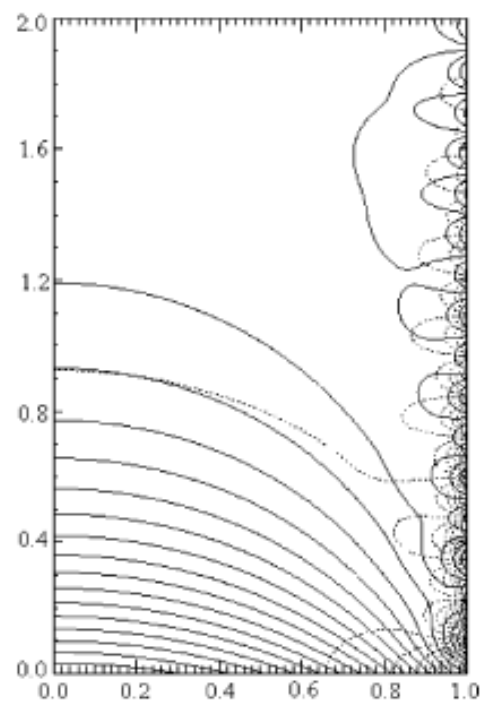

(b)

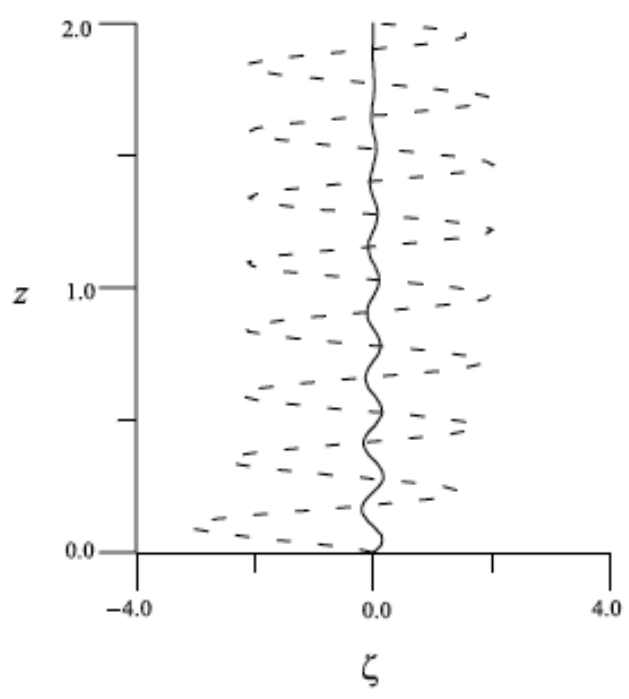

Fig. 4. Isolines of real (solid line) and imaginary (dashed line) parts of potential of pulsating velocity (a), and real (solid line) and inaginary (dashed line) parts of $\zeta(b)$, for We $-15800(w-6000 \mathrm{~s}$ ).

mensions are determined by the wavelength of the surface pulsations. The presence of a propagating component, i.e, non-coincidence of the amplitude of the direct and reflected waves, is, however, of fundamental importance: it leads to the formation of a global mean flow. The intensity of this mean flow is so large that the small vortices are insignificant on its background and become apparent just through a weak additional deformation of the mean free surface.

At frequencies far from the resonant values, the amplitudes of the direct and reflected waves are close to the vibration amplitude, as shown in Figs. $4 \mathrm{~b}$ and 5 where the amplitude of the standing wave is close to 2. However, when the frequency increases, the wavelength decreases. As a result, the pulsating velocity gradient and thus the mean-flow intensity increases. When the vibration frequency approaches one of the fundamental frequencies of the system, the amplitude of surface waves resonantly grows.

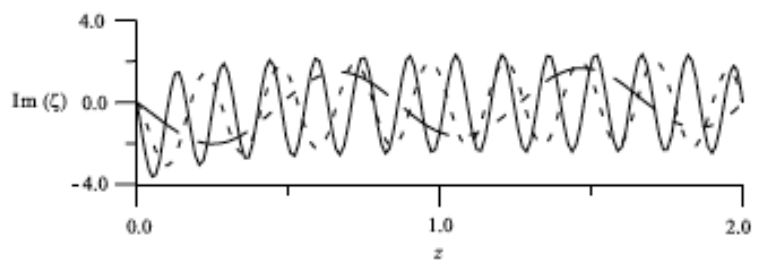

Fig. 5. Imaginary part of dimensionless amplitude of deviation of free surface from its mean shape for $a / R-0.003$ : dash dot line We-439, dashed line $W e-15800$, solid line $W e-64840$ (i.e., $w-1000 s^{1}, w-6000 s^{1}$ and $\omega-12150 s^{3}$, respoctively). 
(a)

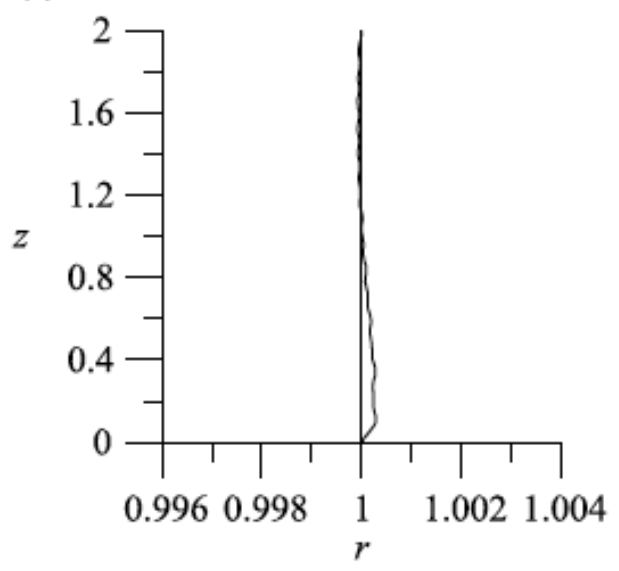

(c)

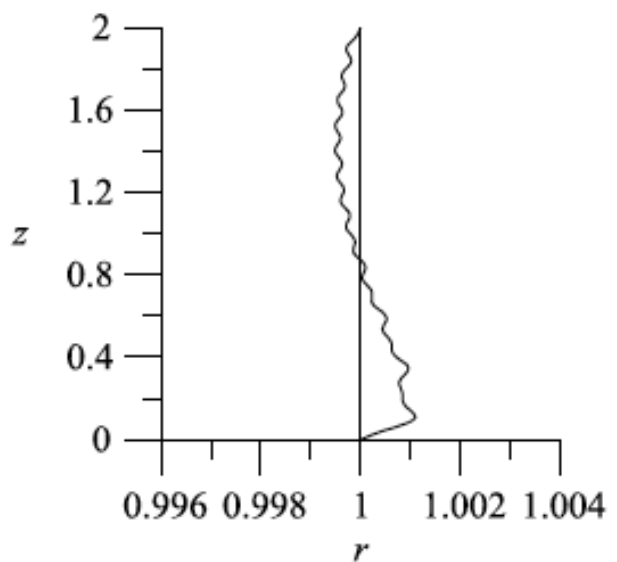

(b)

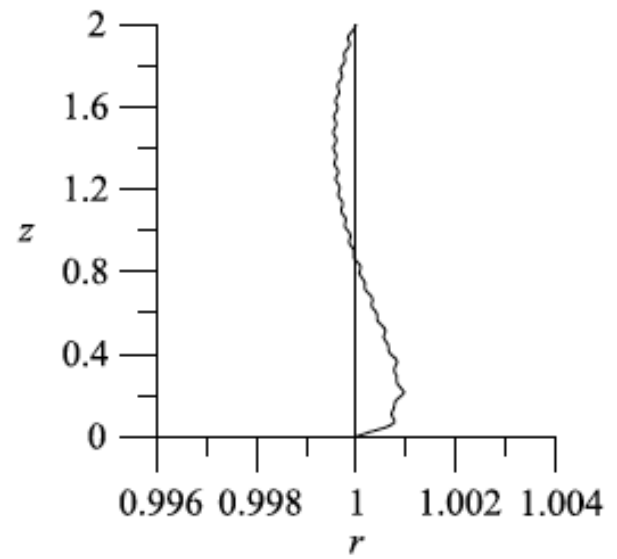

(d)

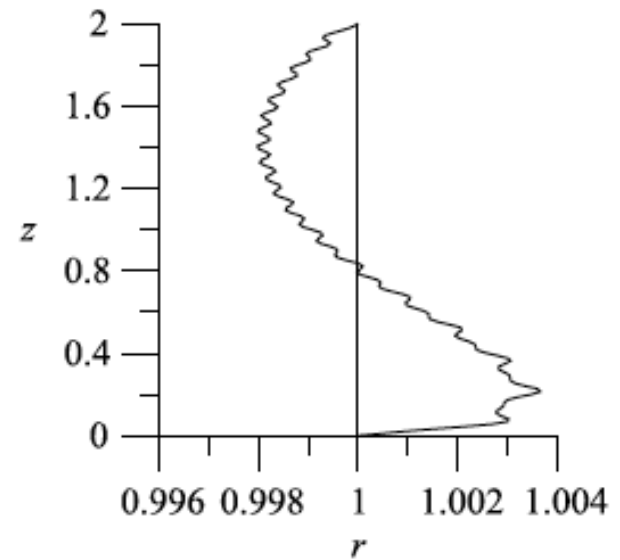

Fig. 6. Mean deformation of the free surface: (a) $a-7.5 \times 10^{4} \mathrm{~cm}, \omega-6000 \mathrm{~s}^{1}(B \approx 0.00889)$; (b) $a-7.5 \times 10^{4} \mathrm{~cm}$, $\omega-12150 \mathrm{~s}^{1}(B \approx 0.0365) ;(c) a-15 \times 10^{4} \mathrm{sm}, \omega-6000 \mathrm{~s}^{1}(B \approx 0.0355) ;$ (d) $a-15 \times 10^{4} \mathrm{~cm}, \omega-12150 \mathrm{~s}^{1}$ $(B \approx 0.146)$.

Fig. 6a d illustrate the influence of vibrations on the mean shape of free surface. By analyzing these figures we can conclude that:

- under the influence of the lower rod vibrations the liquid bridge takes an amphora shape: the free surface is convex near vibrating rod and concave near the rod at rest. This is in agreement with the results [20] concerning the case of stuck-edge conditions on the contact line, where mean shape of a liquid bridge (between two vibrating rods) was calculated by means of variational technique with one trial function;

- mean deformation of free surface is nearly proportional to $(a \omega)^{2}$, i.e. proportional to the vibration parameter $B$. This also well corresponds to the results [20]. The parameter range considered in the present paper (small vibration amplitude and large surface tension coefficient 
typical for liquid metals), corresponds to low values of parameter $B$, for which mean deformations of free surface are small;

- the free sufface exhibits also the small fluctuations (ripple), which reflect the presence of small vortices near the free surface. The spatial period of this ripple is related to the wavelength of the free surface pulsations (see Figs. 5 and 6 for the comparison); it decreases with the increase of the vibration frequency.

The number of nodes in $z$-direction in most of the calculations was taken to be 151 (with an irregular mesh size). This value was chosen on the basis of accuracy test with different node numbers to achieve good convergence at the highest frequency, i.e., $2 \mathrm{kHz}$. In this case, it corresponds to more than 10 nodes per wavelength of the free surface pulsation.

We used a boundary-fitted curvilinear coordinate system, with a mesh refinement near the free surface. The vortex size near the free surface is relatively large in comparison with the mesh size. The characteristic vortex size is defined by the wavelength of free surface pulsation; for the frequency of $1 \mathrm{kHz}$ it is $\sim 0.05$ (see Figs. 4 and 5). The irregular grid in $r$-direction is such that the dimensionless step size varies from 0.0396 (near the symmetry axis) to 0.0055 (near the free surface).

Accuracy tests have been performed for $\omega=6 \times 10^{3} \mathrm{~s}^{-1}$ by using different grids (from $31 \times 121$ to $47 \times 151$ ) and different values of grid-transformation parameters. The flow structure and the free-surface shape remain qualitatively the same. For the extremal streamfunction values, with the same grid-transformation parameters (the ones used in most of the calculations), the difference between the results for the meshes $31 \times 121$ and $47 \times 151$ is of $2.5 \%$. The mean shapes of the free surface obtained in these two calculations are very close and exhibit the same number of ripples (Fig. 7). Some calculations were also carried out with a fixed number of nodes, varying the gridtransformation parameters. The difference in the extremal streamfunction values obtained with

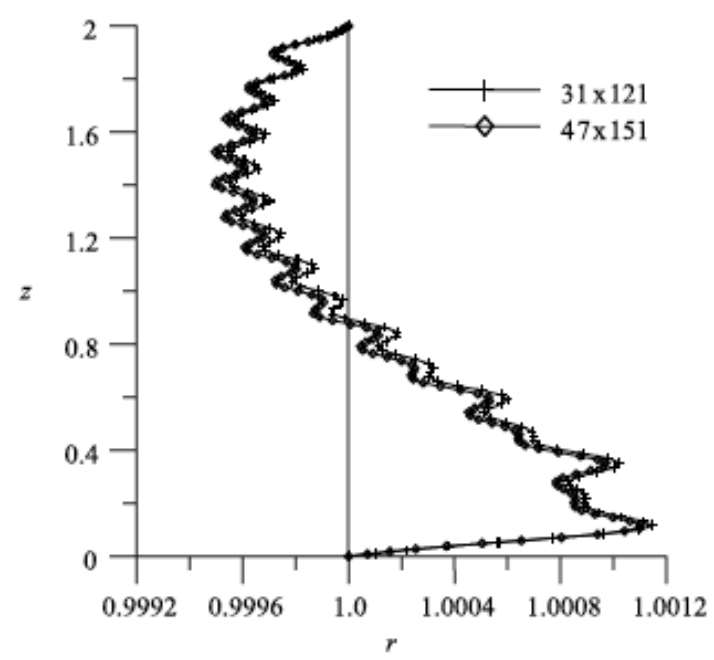

Fig. 7. Mean deformation of the fros surface, for $a-15 \times 10^{4} \mathrm{~cm}$ and $w-6000 \mathrm{~s}^{3}$; computed on two different grids. 
(a)

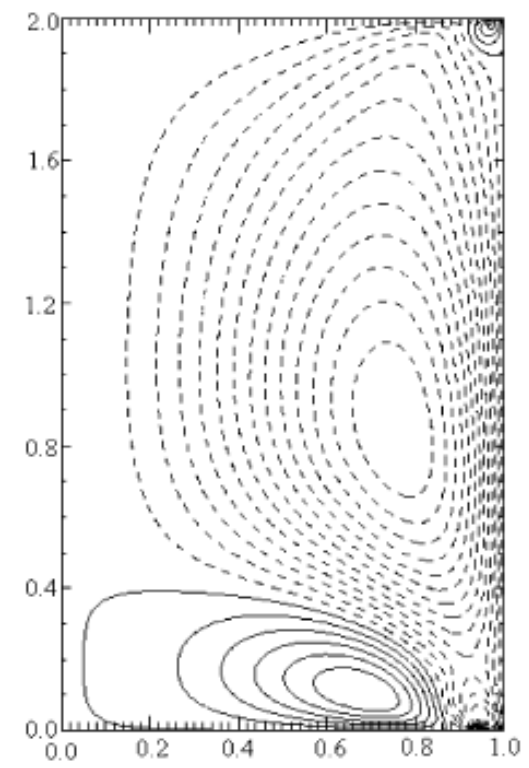

(b)

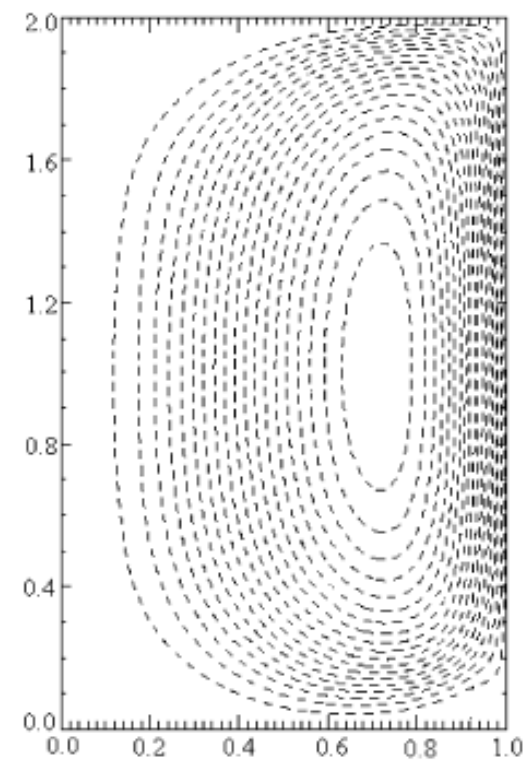

Fig. 8. Isolines of Lagrangian streamfunction of mean flow for $a / R-0.003$ : (a) We $-439\left(w-1000 \mathrm{~s}^{\mathrm{J}}\right)$, $\psi_{\min }-$ $-0.01075, \psi_{\max }-0.00465 ;\left(\right.$ b) $W_{e}-64840\left(w-12150 \mathrm{~s}^{\mathrm{j}}\right), \psi_{\min }--48.59, \psi_{\max }-0.3019$. Dashed lines correspond to negative streamfunction values.

the grid-transformation parameters which generate a virtually uniform grid and those used in most of the calculations is of $3.5 \%$, for the mesh $47 \times 151$.

Furthermore, to test the feedback influence of the surface deformation on the mean flow, we performed additional calculations neglecting the mean deformation. The difference in the minimum values of the Lagrangian streamfunction, accounting for, or neglecting, the mean deformation of the free surface, is very small. So, the mean deformation of the free surface has only a small feedback effect on the mean flow.

Typical views of the Lagrangian streamfunction are presented in Fig. 8a and $b$. They exhibit a dominating vortex, which occupies most part of the liquid zone. This main vortex is induced by surface waves propagating from the lower, vibrating, rod. The direction of this vortex is such that the liquid moves along the free surface from the vibrating rod. The small vortex near the vibrating rod (Fig. 8a) is of Schlichting origin. In this vortex the fluid moves along the rigid surface in the direction opposite to that of the gradient of the pulsating energy, i.e. the flow is clockwise. The Schlichting mechanism is significant only for low values of Weber numbers.

The dependence of the minimal value of the streamfunction (i.e., the maximal value of flow intensity) over the computational domain on the frequency is presented in Fig. 9, in terms of the square root of the Weber number. It shows that the flow intensity increases with the frequency but in a non-monotonous way. The values of the vibration frequency at which a sharp growth of the mean flow intensity occurs well correspond to the resonant frequencies, calculated in Section 3.1. 


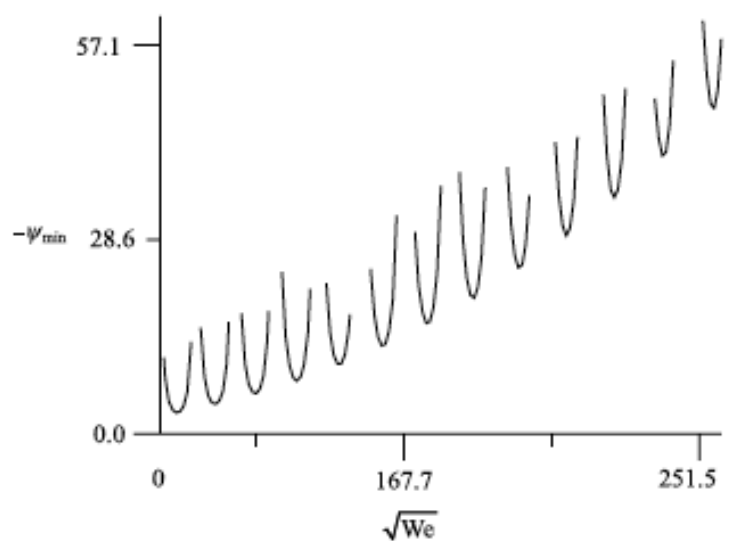

Fig. 9. Dependence of minimal value of streamfunction on We number for $a / R-0.003$.

\section{Conclusions}

With the future goal of demonstrating the feasibility of vibration-driven control of floatingzone growth in Space environment, we have considered a preliminary, more fundamental, study of the vibration-driven flow inside an isothermal liquid bridge limited by two rigid rods with one of the rods subject to high-frequency vibrations in axial direction, under zero gravity conditions, assuming that in the absence of vibrations the liquid bridge has a cylindrical shape. Pulsating and mean flows in an isothermal liquid bridge have been studied, accounting for: (i) meniscus deformation, (ii) generation of mean vorticity in the boundary layers near the rigid and free boundaties, (iii) viscous damping of the surface waves and (iv) spatial inhomogeneity of pulsation phase. Two models have been considered.

In a first model, we assume that the vibrating liquid bridge has a cylindrical shape (i.e., mean deformations of the free surface due to vibrations are ignored). A closed-form solution for the pulsating deformation of the free surface and the pulsating velocity field has been found. The resonant frequencies of the free surface oscillations have been calculated. The solution of the problem for the mean components has been obtained numerically by a finite-difference method.

The second model accounts for the mean deformations of the free surface due to vibrations. The governing equations for the potential of pulsating velocity and mean velocity and for pressure are approximated by using a finite-difference method on a semi-staggered grid. The unknown free surface is determined by using a boundary-fitted curvilinear coordinate system. The resulting non-linear system of algebraic equations is solved by using Newton's method. The vibration influence on the mean shape of the liquid bridge has been analyzed. The mean-flow structure and intensity have been obtained for a wide range of vibration frequency ( $02 \mathrm{kHz}$ ). The mean flow intensity has been found to be a non-monotonous function of vibration frequency. The frequency values where a sharp growth of the mean-flow intensity occurs are in a good agreement with the exact solution of the pulsation problem for a "purely cylindrical" liquid bridge. 


\section{Acknowledgements}

This work has been supported by the Russian Foundation for Basic Research, the Ministry of Education of Russian Federation, the Ministere de l'Education Nationale, de la Recherche et de la Technologie (Division Affaires Internationales) and the European Commission (INCO/ COP-977120 contract).

\section{References}

[1] Anilkumar AV, Grugel RN, Shen XF, wang TG. Control of themocapillary convetion in a liquid bridge by vibration. J Appl Phys 1993;73(9):4165 70.

[2] Shen XF, Anilkumar AV, Grugel RN, Wang TG. Utilizing vibration to promote microstructural homogeneity during floating-zone crystall growth processing. I Crystal Growth 1996;165:438 46.

[3] Zhang Y, Alexander $\Pi$. Sensitivity of liquid bridges subject to axial residual acceleration. Phys Fluids $1990 ; \mathrm{A} 2(1)): 196674$

[4] Nicolas IA, Vega MV. Weakly nonlinear ascillations of nearly inviscid axisymmetric liquid bridges. J Fluid Medh $1996 ; 328.95128$.

[5] La CP, Anilkumar AV, wang TG. Streaming generated in a liquid bridge due to nonlinear ascillations driven by the vibrations of an endwall. Phys Fluids 1996;8(12):3234 46.

[6] Lyubimov DV, Savvina MV, Cherepanov AA. On quasi-equilibrium shape of a free surface in modulated gravity field, The problems of hydromechanics and heat/mass transfer with free boundaries. Novosibirsk 1987. p. 97105.

[7] Lyubimov DV, Cherepanov AA. On the development of steady relief on fluid interface in a vibrational field. Fluid Dym 1987:21:849 54 .

[8] Schlichting G. Grenzschicht-Theory. Karlsruhe: Braun; 1951.

[9] Longuet-Higgins MS. Mass transport in water waves. Phil Trans A 1953;245:535 81.

[10] Lyubinov DV. Convetive flows under the influence of high-frequency vibrations. Eur J Medh, B/Fluids $1995 ; 14(4): 43958$

[11] Lyubinov DV. A new approach in the vibrational convetion theory. CR Acad Sci Paris, Ser IIb 1995,t.320:271 5.

[12] Gershuni GZ, Lyubimov DV. Thermal vibrational convection. London: wiley; 1998.

[13] Lyubinov DV, Lyubimova TP, Roux B. Mechanisms of vibrational control of heat transfer in a liquid bridge. Int I Heat Mass Transfer 1997;40(17):4031 42.

[14] Landau LD, Lifshitz EM. Hydrodynamics. Moscow: Nauka; 1986.

[15] Mason wP, editor. Physical acoustics. New York: Academic Press; 1965.

[16] Lyubinov DV. Thermovibrational flows in nonuniform systems. Mirrogravity Quarterly 1994;4(4)-221 5.

[17] Lyubimov DV, Cherepanov AA, Lyubinova TP, Roux B. Deformation of gas or Arop indusion in high frequency vibrational field. Microgravity Quarterly 1996;6(2 3):69 73.

[18] Ruvinsky KD, Feldstein FI, Freidman GI. Numerical simulations of the quasi-stationary stage of ripple excitation by stexp gravity eapillary waves. J Fluid Mech 1991;30:339 53.

[19] Thompson JF, Thames FC, Mastin CW. I Comput Phys 1974;15:299.

[20] Gershumi GZ, Lyubimov DV, Lyubimova TP, Roux B. Vibrational convection in a heated liquid bridge with free surface. CR Acad Sci, Ser Ilb 1995, t.320:225 30. 